\title{
Is There a Compelling Argument for Ontic Structural Realism?
}

\begin{abstract}
Structural realism first emerged as an epistemological thesis aimed to avoid the so-called pessimistic meta-induction on the history of science. Some authors, however, suggested that the preservation of structure across theory-change is best explained by endorsing the metaphysical thesis that structure is all there is. While the possibility of this latter, 'ontic' form of structural realism has been extensively debated, though, not much has been said concerning its justification. In this paper, I distinguish between two arguments in favour of ontic structural realism that can be reconstructed from the literature, and find both of them wanting.
\end{abstract}

Matteo Morganti

Department of Philosophy

University of Rome 'Roma TRE'

Via Ostiense, 234

00144

Rome

Italy

E-mail:mmorganti@uniroma3.it

Word count: 4967 


\begin{abstract}
Structural realism first emerged as an epistemological thesis aimed to avoid the so-called pessimistic meta-induction on the history of science. Some authors, however, suggested that the preservation of structure across theory-change is best explained by endorsing the metaphysical thesis that structure is all there is. While the possibility of this latter, 'ontic' form of structural realism has been extensively debated, though, not much has been said concerning its justification. In this paper, I distinguish between two arguments in favour of ontic structural realism that can be reconstructed from the literature, and find both of them wanting.
\end{abstract}




\section{Introduction}

The fundamental intuition underpinning scientific realism is expressed by the so-called 'no-miracle argument' (NMA) (Putnam 1975, 73), according to which the success of science is to be explained in terms of the (approximate) truth of scientific theories. ${ }^{1}$ Standard, object-oriented realism has it that successful theories are (approximately) true descriptions of individual objects that exist mind-independently and of their properties. The pessimistic meta-induction on the history of science (PMI) (Laudan, 1981), however, threatens to sever the link between success and truth by pointing to past theories that were successful to some extent but are now considered false.

Structural realism (SR) attempts to re-establish the connection between success and truth by pointing at the structural continuity that exists between (some parts of some) subsequent theories across theory-change. SRists propose that we take (preserved - perhaps via some correspondence principle) structure as the (approximately) true part of such theories. Epistemic structural realism (ESR) (Worrall 1989) intends this as an epistemological position, to the effect that we can be realists about whatever is described by the (preserved) mathematical structure of our theories. Ladyman (1998) introduced instead ontic structural realism (OSR), according to which not only is structure all we can be realist about, but it is also all there is.

\footnotetext{
${ }^{1}$ Whether there is a full-scale argument or just an intuition is open to debate, but this is not important for present purposes.
} 
In the last decade or so, the latter idea has been developed further and has become increasingly fashionable. On the one hand, OSRists argued that 1) it is possible to conceive of the world in structural terms, as there is nothing that remains unaccounted for when one subscribe to a structuralist ontology. On the other hand, OSRists also contended that 2) scientific realists should endorse OSR, as a careful consideration of the best available scientific theories lends stronger support to it than to other non-instrumentalist positions. The discussion of 1) has occupied most of the literature, and it appears fair to say that criticisms aiming to show that the proposed ontology is unworkable don't cause too much trouble for OSRists. Relations, and consequently structures, can be primitive (Mertz 2003) and a plausible structural reconstruction of persistence, change and causality can be provided (French 2003). Moreover, the claim being put forward (in the great majority of cases, at least) is not that the world is mathematical structure, so charges of conflating the mathematical and the physical (Cao 2003) are off the mark. As for the objection that OSR only applies in a very limited field, it has also been answered - by extending OSR to domains other than physics (Ladyman and Ross 2007; Ross 2008; Kincaid 2008). However, much less has been said about 2), that is, about the justification for OSR. To fill this gap, it is essential, first of all, to analyse the relationship between ESR and OSR.

\section{The relationship between ESR and OSR}

One may be inclined to believe that ESR is a stronger claim than OSR on the basis that it requires commitment to structure - i.e., relations - and individuals (possibly, with monadic properties) bearing those relations. Worrall (1989) does indeed echo Poincaré (1905/1952) 
in claiming that relations are all we know, but individual objects - which 'Nature will hide forever from our eyes' - exist in addition to them. However, ESR is a purely epistemological thesis concerning the preservation of structure across theory change and its usefulness for avoiding the PMI. And the Worrall-Poincaré line of thought follows from an adherence to commonsense which is only natural if one refrains in this way from asking metaphysical questions. In other words, ESR does not rely in any way on a 'positive' ontological commitment to the existence of objects. ${ }^{2}$

On the other hand, supporters of OSR have argued that, since the basic assumption underlying SR is that we can know structure only, we should adopt a structuralist ontology if only not to create an unacceptable gap between epistemology and metaphysics. However, setting aside the fact that - to repeat - the realist doesn't need to say anything about metaphysics at all, it is essential to notice that the elements preserved across theory change are (or at least might be) different from those invoked by OSRists. The former are whatever properties (not necessarily limited to polyadic relations!) are described by the mathematical structures that get preserved across theory change. The latter are instead genuine relations, and of a special kind (as we will see in more detail shortly): those that play essential roles in defining specific theoretical descriptions of things, independently of previous theories. ${ }^{3}$ In fact, that the epistemic element and the metaphysical element (may) play distinct roles is something that at least some OSRists openly acknowledge (French

\footnotetext{
${ }^{2}$ This is why it has been suggested (Morganti 2004) that ESR is best understood as agnostic about the existence of individual relata over and above the preserved structure.

${ }^{3}$ This will become clearer in what follows.
} 
2006, 173). Moreover, even assuming that structural preservation suggests a structuralist ontology of the kind envisaged by OSRists, the former is not a sufficient basis for the latter. For, filling the alleged gap between epistemology and metaphysics exclusively on the basis of contingent facts about what got preserved in the history of science may well lead one to ignore important metaphysical elements. In addition to the historical evidence of continuity pointed at to neutralise the PMI, therefore, a consideration of the metaphysical import of our best current theories is also required if the move from the epistemological to the metaphysical level is to be made.

As things stand, then, it seems fair to claim that OSR is in fact stronger and more committing than ESR: for, it amounts to a conjunction of i) an epistemological claim of preservation of structure across theory-change (the 'core' structural realist thesis aimed to defuse the PMI) plus ii) a form of eliminativist metaphysics which is an independent addition to i) in the sense of being justified independently of the evidence coming from the history of science. ${ }^{4}$

\section{The argument from underdetermination}

In the early years of OSR, the argument provided in support of its eliminativist metaphysical component, mainly by French and Ladyman (Ladyman 1998; French and

\footnotetext{
${ }^{4}$ Intermediate, or moderate, versions of SR - according to which relata and relations are (at least in some cases) metaphysically on a par in terms of ontological priority - (see, for example, Esfeld and Lam 2008) gain plausibility only to the extent that OSR does. Hence, these will not be explicitly considered here.
} 
Ladyman 2003), was based on an alleged underdetermination in the ontological interpretation of non-relativistic quantum mechanics. It can be reconstructed as follows:

1. The existence of compelling reasons and of a valid alternative are necessary requisites for the modification of entrenched metaphysical beliefs;

2. Our entrenched metaphysical view of the material world is object-based;

3. Postulating objects as fundamental entities leads to a problematic metaphysical underdetermination (between individual objects and non-individual objects) in the ontological interpretation of quantum mechanics - which is a fundamental theory in current physics, and certainly one that realists need to take into account;

4. A structuralist reconstruction of the quantum domain, according to which objects are not fundamental, is possible;

5. A structuralist reconstruction of material objects gets rid of the underdetermination mentioned in 3 , and thus satisfies 1 ;

C. There are compelling reasons for adopting a structuralist metaphysics. ${ }^{5}$

\footnotetext{
${ }^{5}$ Notice that, while ESR relies on preserved structure in general and can point to particular historical cases, as long as it is justified along the lines being considered here OSR needs instead a specific realist attitude with respect to a specific theory - which, incidentally, is the object of heavy philosophical dispute. Obviously enough, realism about quantum mechanics cannot in turn be based on OSR, on pain of circularity. In view of this, OSR appears parasitic on ESR (other realist positions, we will assume here, having been ruled out on the basis of the PMI). One may complain that one should be allowed to just endorse a generic 'realist stance' and then specify further what form of realism is best justified.
} 
Premise 2 is uncontroversial, and premise 4 has been assumed to be unproblematic in this paper. As for premise 1, it appears quite sensible and at least one respected OSRist openly accepts it (French 2006, 177). What about the other premises?

The claim in 3 has to do with the fact that quantum particles have features that make them strikingly different from classical particles and from the paradigmatic individual objects of everyday life. They do not obey the Leibnizian Principle of the Identity of the Indiscernibles (PII), as they can have all the same properties ${ }^{6}$; and they are 'indistinguishable', so that (very roughly) there are two ways in which two coins can be one heads and the other tails but only one way in which two fermions can be one spin up and the other spin down. It allegedly follows from this that either particles are nonindividuals $^{7}$ - they do not have well-defined identities - or they are individuals whose

However, this would mean to ignore the strength of the PMI, which, by putting the NMA into doubt, makes such a generic realist stance unviable even within realist circles. Hence, ESR must indeed be presupposed by OSRists. (Incidentally, this lends further support to the view of the relationship between ESR and OSR argued for in the previous section).

${ }^{6}$ This claim is in need of qualification, as we will see in the next section. This is immaterial to the reconstruction of the argument from underdetermination.

${ }^{7}$ By which, on what seems to be the most plausible interpretation, one means that they are not basic ontological constituents, but rather 'derivative' manifestations of something more fundamental: for example, one might say that a system of two indiscernible bosons is in fact a bosonic field with a certain 'two-unit' perturbation at a given point. (The more 
individuality is not analysable in terms of qualitative features (and is provided instead by bare particulars, 'haecceitates' or what have you), and whose statistical behaviour is due to some specific non-classical aspect of the relevant domain. ${ }^{8}$ The non-individuality option appears naturally to explain the impossibility of individuation via description or direct reference, and the peculiarity of quantum statistics ('no identities to be permuted'). The individuality view, on the other hand, agrees with commonsense and with the ontological presuppositions that hold true in the classical domain.

According to OSRists, there are no conclusive arguments in favour of either option (rather, problems on both sides), but the metaphysical dilemma can be dissolved altogether by opting for a structuralist perspective that posits relations and not objects as fundamental. ${ }^{9}$ However, consider again the two metaphysical 'packages' just identified. To be sure, each one of them meshes better with certain specific metaphysical theses about objects, properties and identity conditions than with others. And, indeed, it is equally uncontroversial that there is no way to choose one package, with its related set of

fundamental something need not be understood structurally: an ontology without individuals is not ipso facto an ontology of relations only).

${ }^{8}$ This can be achieved in a number of ways (none of which absolutely uncontroversial, of course): for a discussion of them - and a proposal - see Morganti 2009.

${ }^{9}$ Essentially because, if objects are derivative on structures, the peculiar features they exhibit in the quantum domain need not be explained against a more or less traditional ontological background, and can instead be attributed directly to the more fundamental structural - level. 
metaphysical theses, rather than another exclusively on the basis of the available data. But this is not a problem for the typical metaphysician! For, far from expecting to be able to extract well-defined metaphysical theses from the best available scientific descriptions of things, s/he always anticipates that this is normally not the case, and other factors will have to be brought to bear. As a matter of fact, the typical metaphysician starts from certain metaphysical views - arrived at on mostly a priori grounds - and evaluates how these fare with respect to a range of considerations including, but not reducing to, an assessment of how those views fit with the input coming from the actual world. In view of this, it seems fair to say that, in the case under scrutiny, metaphysicians (at least those who have clear opinions about the constitution of material objects and their individuation) would simply disagree with the claim that there is a problematic underdetermination. An ontological account of the relevant domain, they would argue, can be provided in the framework of either one of the two object-based ontologies - one just needs to be clear about the assumptions needed to do this (and the consequences of each interpretation). ${ }^{10}$

A sensible reply to this is that a priori considerations never provide us with firm grounds for choosing one set of assumptions over others, and so we can (and should) only do metaphysics a posteriori, so dismissing traditional metaphysical arguments altogether. That this is what OSRists think is suggested by the fact that in the extant formulations of the

${ }^{10}$ More generally, from the point of view of traditional metaphysics there doesn't seem to be more underdetermination in quantum mechanics than in any other domain of reality that we attempt to characterise in metaphysical terms. On this point, see (Chakravartty 2007, 74-75). 
argument under consideration they just state that there are different object-based accounts of the particles' nature, and do not engage a critical examination. The problem with this reply, though, is that the OSRist claim being assessed is simply that standard objectoriented realism, based as it is on traditional metaphysical presuppositions, meets with an insurmountable problem of underdetermination when it comes to interpreting quantum mechanics. At no point are methodological considerations about what counts as 'proper' metaphysics brought into play.

In light of the above, it appears legitimate to reject premise 3 above. But let us accept that premise for the sake of argument. At least two interrelated questions are left outstanding: isn't the structuralist option also underdetermined by the available evidence? Why exactly should one think that it provides a way out of the underdetermination being pointed at? As for the first question, perhaps the thought is that the structuralist view is not a third option, but rather a sort of 'synthesis' between the individuality and the non-individuality packages. That this is not the case, however, appears quite clear: both the individuality and non-individuality views are based on objects and monadic properties (possessed by or constituting the former) as fundamental; consequently, relations do not constitute a 'common core' between the two. Moreover, if a synthesis is what one is looking for, there is a large number of properties that are shared by the two accounts, and about which a realist can be realist without also taking a stance regarding the nature of objects in general certainly without being led to take only relations seriously. ${ }^{11}$

${ }^{11}$ Saatsi (2010) voices similar doubts. The OSRist may argue that, unlike the 'selective sceptic' (see Chakravartty 2003, 2007) who restricts his/her realist commitment to some of 
For what concerns the second question, OSRists often say that one should re-conceptualise the quantum domain in terms of physical structures as primitives, from which objects 'emerge' as relational 'nodes' (French 2006, 183). But (even) if objects emerge, don't we have to account for their (non-)individuality anyway? Compare with mathematical structuralism. Even once each element, say, in the structure of natural numbers is defined purely structurally, every natural number has definite identity conditions. Therefore - at least relative to one's assumptions about what being an individual exactly means - one is in a position to state whether natural numbers are individual or non-individual objects. What about physical objects? The only reaction available to OSRists seems to be to regard the latter as entirely 'epiphenomenal', and thus not requiring (nor permitting) any metaphysical elucidation.

This is enough to suggest that it is far from clear that structuralism is the most natural and least costly solution to the (alleged) problem of underdetermination. In light of this, one may legitimately put premise 5 above into doubt.

More generally, there are grounds for contending that the argument for OSR based on the (alleged) underdetermination of metaphysics by non-relativistic quantum mechanics is unsound, and thus cannot constitute the basis for a compelling justification of OSR.

the properties of things while sticking to a 'canonical' understanding of the latter, the structuralist can focus more directly on these properties as they emerge from the theory itself. This, however, presupposes the plausibility of the ontic structuralist realist view, which is clearly different from a generic property-based realism. 


\section{The argument from the primacy of relations}

If the arguments presented in the previous section are correct, merely 'negative' considerations against other forms of realism (based on the alleged shortcomings of the latter with respect to the interpretation of physical theory) are not sufficient for a persuasive justification of OSR. Consequently, the OSRist will have to provide direct, 'positive' support for his/her structuralist ontology. This leads us to the critical assessment of a second argument for OSR that can be found in the recent literature.

It has been contended (Saunders 2006; Muller and Saunders 2008; Muller and Seevinck 2009) that quantum particles are not discernible on the basis of the 'canonical' versions of PII - which only quantify over monadic properties - but are in fact discerned 'weakly' by non-supervenient, irreflexive and symmetric relations such as 'has opposite spin to' and, consequently, via some Leibnizian principle that takes relations of this type into account. At least once one accepts that metaphysically genuine relations need not be reducible to monadic properties nor depend on the existence of already well-defined relata, such weak discernibility of quantum particles entails that the non-individuality option above is untenable. This, in turn, may seem to mean that the underdetermination is broken and, as a consequence, OSR is undermined. However, Muller (forthcoming) contends that only the first conjunct of this latter claim is true: for, objects which are only weakly discernible entirely depend on relations for their identity, and such relationals fit the structuralist picture perfectly well. In fact, Muller argues, the 'discovery' that quantum particles are relationals is very good news for the OSRist. 
This, of course, provided that not only the identity-determining factors but all properties are shown to be (reducible to) relations.

It doesn't come as a surprise, then, that a structuralist analysis of all physical properties has been undertaken by several philosophers, with the aim of showing that objects and monadic properties are 'idle' and play no role whatsoever in physical theory, and there consequently are good reasons for believing that nothing exists over and above structure. ${ }^{12}$ Notice that, quite importantly, what must be shown for this argument to be effective is not only that all properties of things depend on things other than those exemplifying them (i.e., that no property is intrinsic and non-relational), but also that - more strongly - every property is a relation.

OSRists customarily (French 1999, 2003; Ladyman 2009, Sec. 4.1) quote or mention historical figures such as Cassirer, Born, Weyl and Eddington as authoritative exponents of the view that objects coincide with the identification of invariants with respect to the mathematics relevant to the theory; and then connect these historical remarks to more theoretical considerations. Muller (forthcoming), for example, argues that the (allegedly) monadic and intrinsic (essential) properties of quantum particles are in fact invariants of the symmetry groups that define the qualitative features of quantum mechanical systems, and are consequently reducible to the relations that constitute those groups. ${ }^{13}$ Others

\footnotetext{
${ }^{12}$ In particular, positive arguments coming directly from physics are taken by OSRists to ground, together with historical considerations, a sort of 'consilience' in favour of their position (Ladyman and Ross 2007, 161).

${ }^{13}$ See also (Castellani 1998) and (French 2000).
} 
(Kantorovich 2003; Lyre 2004) argue in analogous fashion that in gauge quantum field theories objects are secondary to structure because symmetries are fundamental in the constitution of fields. In general, OSRists interpret the mathematical nature of physical theory and the fact that all physical properties must be 'extracted' somehow from symmetries and invariants that can be traced in the formalism of the theory as clear signs that the things described by the theory and their properties are themselves reducible to the relations that define such symmetries and invariants.

Now, whether or not this way of drawing metaphysical consequences from features of the language that we use to describe things is considered acceptable - a complex issue we will not discuss here -, it seems undeniable that the claims made by OSRists in the present context are considerably unclear. What does it mean, exactly, that objects and properties are/reduce to invariants, or that symmetries are ontologically prior to objects? As a matter of fact, it looks like an important distinction is overlooked (or consciously ignored) here between the formal definition of general, abstract properties on the one hand and the concrete property-instances that exist in the material world on the other. Indeed, the claim, say, that objects and properties can be picked out via the identification of invariants across transformations in the groups that define the relevant theoretical structure seems to rest on a fatal conflation of these two elements. When one focuses on invariants and the likes, one moves at a high level of abstractness, involving the 'general properties of the general properties', so to put it, of types of things. Object- and property-tokens can certainly not be 'found' there. For instance, one can say (with Weyl) that all quantum numbers are indices characterising representations of groups; but while this provides the 
basis, for instance, for distinguishing fermions and bosons at the abstract theoretical level, it doesn't in any way ground a reduction of the actual properties of an actual fermion or boson, which (perhaps with the exception of spin) simply are not the immediate referents of those indices. Thinking otherwise would be like, say, expecting the actual causal features shared by coloured material things to be reducible to the general features shared by abstract concepts and words such as 'Redness', 'Greenness' etc. ${ }^{14}$

Note that the difference involved here is a difference of category: on one side, there are the properties of the abstract, formal structures used to describe the physical world, on the other the properties of concrete things. Consequently, it won't do for the OSRist to just claim that the specific property-instances of specific actual objects can be extracted via a structural analysis of the theoretical apparatuses employed for describing individual physical systems ${ }^{15}$, for that would (perhaps) deflate the general/particular distinction, but there would still be a gap between the concrete and the abstract.

The only remaining option for OSRists is to refuse the request for philosophical analysis on the basis that there is no need to explain how the physical is to be extracted from the abstract. But this can only mean either that i) the physical is eliminated and abstract structure is directly invested with the role of ontologically fundamental entity; or ii) that

14 Incidentally, notice that the 'indices characterising representations of groups' are something different from the general relations peculiar to those groups.

${ }^{15}$ For instance, by moving from global symmetry groups that apply to entire fields to localised groups describing local fields. 
the abstract and the concrete are indeed distinct, but the specific way in which they are related is sufficiently graspable for OSR to be compelling without further elaboration. Option i) amounts to a form of realism about mathematical, not physical, structure (for something going in this direction, see Tegmark 2007). Beside having been ruled out at the outset here, such a view is certainly regarded as a minority view, and one that is more revisionary than one would hope, among OSRists themselves. As for ii), it seems to be the view underlying, for instance, Ladyman and Ross' refusal to say anything about what makes a structure physical rather than mathematical $(2007,158)$. However, such a refusal will hardly appear motivated to those not already convinced by OSR. To be sure, any revisionary thesis bears the burden of proof, i.e., needs to be supported by explicit arguments when it comes to a comparative assessment.

Nor is the case for OSR made any stronger by a further consideration of contemporary physics. Muller, for example, mentions results related to relativistic quantum field theories (the 'Casimir effect', showing that the physical vacuum contains no particles but is not devoid of physical activity; and existing proofs to the effect that both the number and the localizability of particles become frame-dependent in a relativistic context) to conclude that objects really find no room in our best science anymore, and we should consequently make do with relations only. However, to what extent do these results put into question the notion of an object rather than 'just' the concept of a classical (i.e., well-localised etc.) object? Or, consider space-time physics: while the hole argument and related considerations certainly represent a problem for the naïve substantivalist, it is far from 
generally agreed that a structuralist understanding of space-time is the most plausible option in view of them.

In conclusion, then, the argument for OSR based on the (alleged) priority of relations over objects and monadic properties - witnessed by contemporary physics - can also be deemed unsatisfactory.

From this and what was said in the previous section, it can be concluded that both arguments provided so far in support of OSR are unconvincing. In fact, once they are appropriately disentangled and analysed, it can be seen that several considerations are often run together based on the erroneous feeling that they complement and strengthen each other. This gives the wrong impression that a full-blown argument for OSR has been provided. To the contrary, OSR may well be a possible realist position, but it is far from clear that it has been supplied with a compelling justification and, therefore, that it truly is the most forceful option available to the scientific realist. 


\section{References}

Cao, Tian Y. 2003. "Structural Realism and the Interpretation of Quantum Field Theory." Synthese 136: 3-24.

Castellani, Elena. 1998. "Galilean Particles: An Example of Constitution of Objects." In Interpreting Bodies: Classical and Quantum Objects in Modern Physics, ed. Elena Castellani, 181-194. Princeton: Princeton University Press.

Chakravartty, Anjan. 2003. "The Structuralist Conception of Objects.” Philosophy of Science 70: 867-878.

Chakravartty, Anjan. 2007: A Metaphysics for Scientific Realism: Knowing the Unobservable. Cambridge: Cambridge University Press

Esfeld, Michael, and Vincent Lam. 2008. "Moderate Structural Realism about SpaceTime." Synthese 160: 27-46.

French, Steven. 1999. "Models and Mathematics in Physics: The Role of Group Theory." In From Physics to Philosophy, ed. Butterfield, Jeremy and Constantine Pagonis, C., 187-207. Cambridge: Cambridge University Press.

French, Steven. 2000. “The Reasonable Effectiveness of Mathematics: Partial Structures and the Application of Group Theory to Physics." Synthese 125: 103-120. French, Steven. 2003. “Scribbling on the Blank Sheet: Eddington's Structuralist Conception of Objects." Studies in History and Philosophy of Modern Physics 34: $227-259$.

French, Steven. 2006. "Structure as a Weapon of the Realist." Proceedings of the Aristotelian Society, 106: 167-185. 
French, Steven, and James Ladyman. 2003.” Remodelling Structural Realism: Quantum Physics and the Metaphysics of Structure." Synthese 136: 31-56. Kantorovich, Aharon. 2003. "The Priority of Internal Symmetries in Particle Physics." Studies in History and Philosophy of Modern Physics 34: 651-675.

Kincaid, Harold. 2008. "Structural Realism and the Special Sciences.” Philosophy of Science 75: 720-731.

Ladyman, James, and Don Ross (with David Spurrett and John Collier). 2007. Every Thing Must Go: Metaphysics Naturalised. Oxford: Oxford University Press. Ladyman, James. 1998. “What is Structural Realism?” Studies in History and Philosophy of Science 29: 409-424.

Ladyman, James. 2009. “Structural Realism.” In Stanford Encyclopedia of Philosophy, ed. Zalta, Edward N., (Summer 2009 Edition), URL = <http://plato.stanford.edu/archives/sum2009/entries/structural-realism/>. Laudan, Larry. 1981. “A Confutation of Convergent Realism.” Philosophy of Science 48: 19-49.

Lyre, Holger. 2004. "Holism and Structuralism in U(1) Gauge Theory." Studies in History and Philosophy of Modern Physics 35: 643-670.

Mertz, Donald W. 2003.”An Instance Ontology for Structures: Their Definition, Identity, and Indiscernibility." Metaphysica 4: 127-164.

Morganti, Matteo. 2004. "On the Preferability of Epistemic Structural Realism." Synthese 142: 81-107. 
Morganti, Matteo. 2009. "Inherent Properties and Statistics with Individual Particles in Quantum Mechanics. " Studies in History and Philosophy of Modern Physics 40: 223-231.

Muller, Fred A. Forthcoming. "Whithering Away, Weakly.” Synthese.

Muller, Fred A., and Simon Saunders. 2008. "Discerning Fermions.” British Journal for the Philosophy of Science 59: 499-548.

Muller, Fred A., and Seevinck, Michael P. 2009. "Discerning Elementary Particles." Philosophy of Science 76: 179-200.

Poincaré, Henry. 1905/1952. Science and Hypothesis. New York: Dover.

Putnam, Hilary. 1975. Mathematics, Matter and Method. Cambridge: Cambridge University Press.

Ross, Don. 2008. "Ontic Structural Realism and Economics.” Philosophy of Science 75: 731-741.

Saatsi, Juha. 2010. "Whence Ontic Structural Realism?” In EPSA. Epistemology and Methodology of Science.Launch of the European Philosophy of Science Association, ed. Suarez, Mauricio, Mauro Dorato, and Miklos Redei, 255-266. Dordrecht, Heidelberg, London, New York: Springer.

Saunders, Simon. 2006. “Are Quantum Particles Objects?” Analysis 66, 52-63.

Tegmark, Max. 2007. “The Mathematical Universe.” Foundations of Physics 38: $101-150$.

Worrall, John. 1989. "Structural Realism: The Best of Both Worlds?" Dialectica 43: 99-124. 